\title{
Directional optical coherence tomography reveals melanin concentration-dependent scattering properties of retinal pigment epithelium
}

Ratheesh K. Meleppat

Pengfei Zhang

Myeong Jin Ju

Suman K. Manna

Yifan Jian

Edward N. Pugh, Jr.

Robert J. Zawadzki 


\title{
Directional optical coherence tomography reveals melanin concentration-dependent scattering properties of retinal pigment epithelium
}

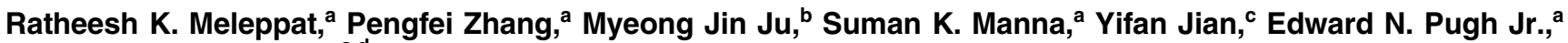 \\ and Robert J. Zawadzki, ${ }^{a, d, *}$ \\ aUniversity of California Davis, UC Davis Eyepod, Department of Cell Biology and Human Anatomy, Davis, California, United States \\ ${ }^{b}$ Simon Fraser University, School of Engineering Science, Burnaby, British Columbia, Canada \\ 'Oregon Science and Health University, Casey Eye Institute, Portland, Oregon, United States \\ dUniversity of California Davis, UC Davis Eye Center, Department of Ophthalmology and Vision Science, Sacramento, California, \\ United States
}

\begin{abstract}
Optical coherence tomography (OCT) is a powerful tool in ophthalmology that provides in vivo morphology of the retinal layers and their light scattering properties. The directional (angular) reflectivity of the retinal layers was investigated with focus on the scattering from retinal pigment epithelium (RPE). The directional scattering of the RPE was studied in three mice strains with three distinct melanin concentrations: albino (BALB/C), agouti (129S1/SvlmJ), and strongly pigmented (C57BL/6J). The backscattering signal strength was measured with a directional OCT system in which the pupil entry position of the narrow OCT beam can be varied across the dilated pupil of the eyes of the mice. The directional reflectivity of other retinal melanin-free layers, including the internal and external limiting membranes, and Bruch's membrane (albinos) were also measured and compared between the strains. The intensity of light backscattered from these layers was found highly sensitive to the angle of illumination, whereas the inner/outer segment (IS/OS) junctions showed a reduced sensitivity. The reflections from the RPE are largely insensitive in highly pigmented mice. The differences in directional scattering between strains shows that directionality decreases with an increase in melanin concentrations in RPE, suggesting increasing contribution of Mie scattering by melanosomes. $\odot$ The Authors. Published by SPIE under a Creative Commons Attribution 4.0 Unported License. Distribution or reproduction of this work in whole or in part requires full attribution of the original publication, including its DOI. [DOI: 10.1117/1.JBO.24.6.066011]
\end{abstract}

Keywords: directional scattering; melanin; optical coherence tomography; retinal imaging; retinal pigment epithelium.

Paper 190040R received Feb. 21, 2019; accepted for publication May 30, 2019; published online Jun. 28, 2019.

\section{Introduction}

Over the past several years, optical coherence tomography (OCT) has evolved into a powerful tool for the in vivo investigation of cross-sectional retinal anatomy and pathology. ${ }^{1,2}$ OCT extracts depth-resolved information about the intensity of backscattered light from retinal layers using low coherence interferometry. The amount of light backscattered/reflected from the retinal layers can provide critical information regarding tissue properties and underlying changes. Furthermore, measuring changes in the angular (directional) scattering of retina layers might further enhance our ability to sense microscopic changes in cellular and subcellular morphology that might precede and/ or follow disease progression. However, this information is not utilized in current clinical OCT systems. The reduction in light scattered by the retinal nerve fiber layer (RFNL) near the optic nerve head is reported to be an early indicator of axonal degeneration and a sensitive way to detect glaucomatous damage. ${ }^{3-5}$ The reflection from the RFNL is found to be highly directional, and the implications of the variability caused by the directional reflectance on clinical measurements are reported. ${ }^{6}$ The directional scattering properties exhibited by the Henle's fiber layer

*Address all correspondence to Robert J. Zawadzki, E-mail: rjzawadzki@ ucdavis.edu
(HFL) in human retina account for their oblique orientation with respect to the outer nuclear layer (ONL). Therefore, directional OCT (dOCT) imaging of human retina enables the delineation of HFL from the ONL and accurate measurement of macular photoreceptor nuclear layer thickness, which are important biomarkers for retinal degenerations. ${ }^{7,8}$ The directional reflectance property shown by the photoreceptor bands, known as the optical Stiles-Crawford effect (SCE), provides the information on the structural integrity of the photoreceptors. An OCT-based assessment of the reflectivity of the photoreceptors layer is reported to study SCE in human retina. ${ }^{9}$

The evaluation of the RPE in vivo has great significance in both visual science and clinical studies, since RPE health will directly affect photoreceptor-RPE-choroid neurovascular unit (PRC-NVU) function. PRC-NVU plays a key role in the process of the vision-sustaining visual cycle and contributes to photoreceptor pigment regeneration. The role of RPE melanin as a major protective agent in the RPE cells sheltering the RPE from exposure to radiation, oxidative stress, and light damage is well documented. ${ }^{10-12}$ Ultrastructural studies have shown that RPE melanosomes undergo significant changes with age or disease. ${ }^{13-15}$ A noninvasive in vivo assessment of melanin changes in the RPE could be a valuable adjunct in the clinical diagnosis of aging and disease progression. Near-infrared autofluorescence (NIA) has been used to visualize the melanin 
distribution in human outer retina. ${ }^{16,17}$ However, overall NIA emission intensity is contributed by the melanin distribution in the RPE as well as in choroid. Therefore, it is practically impossible to differentiate the contribution from melanin in the RPE alone by using simple fluorescent intensity detection.

OCT enables the delineation of retinal layers at cellular resolution and allows the measurement of backscattered light from individual layers. The presence of melanin causes increased backscattering from the RPE and appears as a hyper-reflective band in clinical OCT images. Owing to high melanin scattering, the RPE appears as a thick bright band that can obscure adjacent layers. ${ }^{18}$ Thus, RPE melanin scattering limits the visibility of Bruch's membrane (BrM) and rod outer segment tips, as well as cone outer segment tips in the human fovea, in OCT images. The relative levels of melanin in different individuals could be potentially derived from the changes in the reflectivity of the RPE layer or from the visibility of adjacent layers. However, the accuracy of this approach is often limited by several factors, including system axial and lateral resolution and position of the focal plane, OCT sensitivity roll-off, acquisition settings, and intersubject variability. ${ }^{18}$ The ability of RPE melanin to depolarize the backscattered light enables polarization-sensitive OCT (PSOCT) to segment RPE layer and study melanin-related changes in RPE. ${ }^{19,20}$ In addition to the investigation in animal models, this feature of PSOCT has also been also used in clinical studies of agerelated macular degeneration, RPE detachment, and pseudovitelliform dystrophies. ${ }^{21,22}$ Photothermal OCT (PTOCT) is another functional extension of OCT, based on the photothermal effect, which enables the detection of the optical absorbers in tissues. By taking advantage of the strong absorption in IR by melanin, Lapierre-Landry et al. $^{23,24}$ demonstrated the capability of PTOCT to image melanin distribution in mice and zebrafish retina, and the migration of melanin within RPE in response to dark and light adaptation.

Recently, the potential of angle-resolved measurement of the backscattered signals to provide information on the underlying chemical and physical changes of tissue components has been reported. ${ }^{25-27}$ This approach is based on the acquisition of signals from different backscattering angles and separation of the detection path from the illumination. dOCT is a variant of angle-resolved OCT where both illumination and detection path remain the same, and the imaging is performed with a rotating/translating imaging probe.

In this investigation, we show that the magnitude and directional scattering from the RPE measured with a dOCT correlates with the melanin concentration in the RPE, and thus can potentially serve as an indicator of pigmentation (melanin) level in RPE cells. A custom-built dOCT system was used to investigate in vivo the directional reflectivity of RPE and other reflective (melanin-free layers) in mice retina. The dOCT imaging was performed with a swept source-based OCT (SS-OCT) system operating at a center wavelength of $1060 \mathrm{~nm}$. Three mice strains, modeling different concentration of melanin: melanin-lacking albino (BALB/c), strongly pigmented (C57BL/6J), and mildly pigmented agouti (129S1/SvlmJ) mice were used for the study. The dOCT images were acquired by varying the position of the entrance pupil (imaging beam) with respect to the dilated mouse pupil using motorized linear stages. The reflectivity of each layer was measured from average A-scan profiles and the angular dependence of the intensity quantified with Gaussian profiles fitted to the experimental data.

\section{Materials and Methods}

\subsection{Swept-Source OCT-Based dOCT System}

A schematic representation of the custom SS-OCT-based dOCT system is shown in Fig. 1. The swept source laser (Axsun Technology, Massachusetts) has a center wavelength of $1060 \mathrm{~nm}$ and tuning range of $100 \mathrm{~nm}$. The axial resolution (in air) and peak sensitivity of the SS-OCT are $7.5 \mu \mathrm{m}$ and $101 \mathrm{~dB}$, respectively. The incident power of the imaging beam on the mouse eye was measured to be $\sim 1 \mathrm{~mW}$. The optical configuration of OCT interferometer comprises three 50/50 couplers. ${ }^{28,29}$ The light from the swept source is directed to the interferometer coupler (FC2) via the first coupler (FC1). The two output ports of the coupler FC2 constitute the two arms of a Michelson interferometer. In the reference arm, the light is collimated and projected onto a reference mirror through a dispersion compensator and achromatic lenses, whereas in the sample arm, the probe beam is scanned over the retina using a galvanometric mirror-based beam steering setup (Cambridge Technology, Massachusetts). A scanning lens (L3) and a tube lens (L4) deliver a collimated beam with diameter of $\sim 400 \mu \mathrm{m}$ on the mouse cornea. The reflected light from both interferometric arms is combined at the coupler and the resulting spectral interferogram is detected by a $350-\mathrm{MHz}$ balanced photodetector (PDB430C, Thorlabs Inc.) and sampled by a digitizer (ATS9350, AlazarTech Inc.). ${ }^{30,31}$ The galvanometric mirror scanning unit is synchronized with the data acquisition system controlled by a DAQ Card (PCI6202, National Instruments).

A high-performance computational platform based on CUDA-enabled GPU (Nvidia GetForce 980-gtx) is used to perform real-time processing of interferometric data and image display. This allows the efficient positioning and imaging of the mouse eye without the use of a pupil camera. High-speed imaging enables acquisition of the three-dimensional OCT data well before the mice eyes are affected by cataract. The imaging probe of OCT system is mounted on an $X-Y-Z$ motorized stage (Zaber Technologies, Canada) allowing the controlled and automated positioning of the imaging beam with respect to the dilated mouse pupil, as shown in Fig. 1.

\subsection{Mouse Handling}

All mouse husbandry and handling, including imaging, were in accordance with an animal study protocol approved by the University of California Animal Care and Use Committee, which is accredited by Association for Assessment and Accreditation of Laboratory Animal Care International and strictly adhere to the guidelines set forth by the National Institutes of Health (NIH) and Association for Research in Vision and Ophthalmology (ARVO). The right eyes of four albino (BALB/c, 1-year-old, female), four pigmented (C57BL/6J, 1-year-old, female), and four agouti (129S1/SvlmJ, 1 -year-old, female) were imaged for the study. All mice strains were obtained from the Jackson Laboratory (Sacramento, California). The mouse was positioned on a custom-made platform, and body temperature was maintained and monitored with a heating pad interfaced with a temperature controller. A bite bar and metallic nose mask attached to the platform allowed the stable positioning of the mouse head. During image acquisition, mice were anesthetized with the inhalational anesthetic isoflurane $\left(2 \%\right.$ in $\left.\mathrm{O}_{2}\right)$. Pupils were dilated with $1 \%$ tropicamide and $2.5 \%$ phenylephrine (Akorn, Inc., Lake Forest, Illinois). 

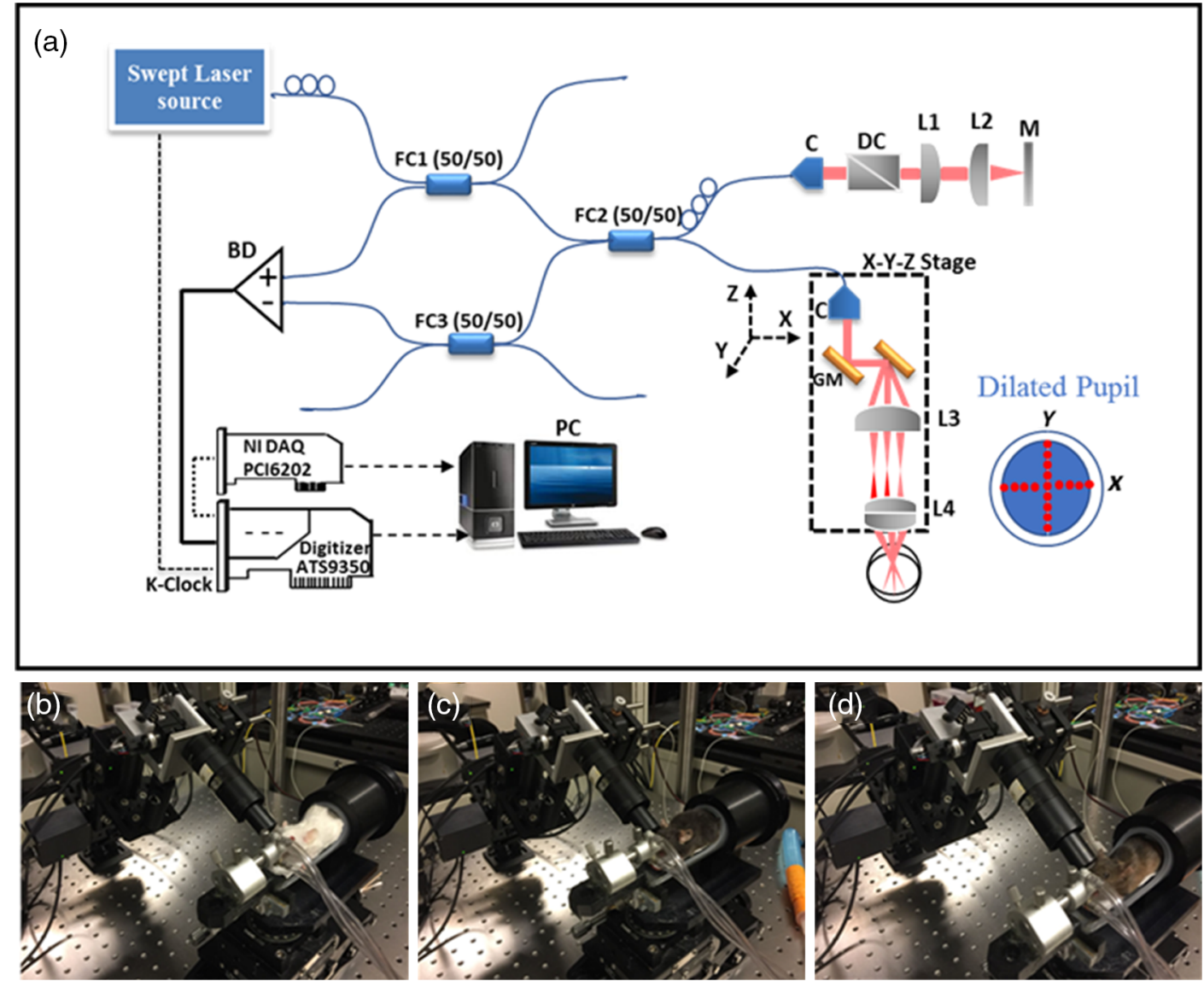

Fig. 1 (a) Schematic representation of source-based dOCT system. BD, Balance detector; C, coupler; DC, dispersion compensator; FC, fiber coupler; L1-L2, Achromat; L3, scanning lens; L4, tube lens; and GM, Galvo mirror. Blue lines-fibers; black lines electrical wires, red lines-free space light path. (b)-(d) Picture of OCT probe with translation stage during imaging session for (b) albino (c) strongly pigmented (d) agouti mice.

To keep the cornea hydrated, lubricating eye drops (Z-drop Vet PLUS, Canada) were supplied to the cornea at regular interval throughout the duration of imaging. No corneal contact lens was used during imaging sessions in these studies.

\subsection{Image Acquisition and Measurement Procedure}

The dOCT imaging was performed by laterally shifting the entrance pupil position (imaging beam) along horizontal $(H)$ and vertical $(V)$ directions across the dilated mouse eye pupil, as shown in Fig. 1. The entrance pupil position was shifted to a maximum of $800 \mu \mathrm{m}$ on either side of the pupil center with a step of $100 \mu \mathrm{m}$. At each pupil position, the OCT system collected five closely spaced ( $2 \mu \mathrm{m}$ each) B-scans at $20^{\circ}$ field of view (FOV) corresponding to a patch of the retina of size $\sim 10 \mu \mathrm{m} \times 680 \mu \mathrm{m}$. The number of B-scans acquired at each retinal location was set to three, so a total of $15 \mathrm{~B}$-scans were acquired. An inter-frame motion correction algorithm was applied to all B-scans using a custom MATLAB Script. The $15 \mathrm{~B}$-scans acquired for each imaging beam position were registered and averaged using Image $\mathrm{J}$ (Turbo Reg). ${ }^{32}$ The lateral displacement of the OCT beam at the pupil plane resulted in a tilt of the B-scans (see Sec. 5). The averaged B-scans from all beam entry positions were tilt-corrected using affine transform and registered using Image J (Stack Reg). ${ }^{32,33}$ A total of 20 well- aligned intensity A-scans were selected and averaged from each averaged B-scans for further analysis. The reflections from the retinal layers measured by OCT are affected by attenuation of the light passing through the ocular media (cornea, lens, and vitreous) due to local variation in absorption or scattering and may change with pupil position. ${ }^{34,35}$ There is also a possibility of second-order intensity variations due to transverse chromatic aberration. ${ }^{36}$ The temporal fluctuations in instrument performance (sensitivity) could also affect overall reflectance measurement. The ONL is a uniformly scattering layer of the retina due to the round shape of nuclei in a watery cell body and is not sensitive to directional illumination. Thus, any change in intensity fluctuations caused by the aforementioned reasons would be reflected in the average intensity (background) of the ONL. Normalization of the A-scans intensity to ONL reflection corresponding to different pupil entry positions will nullify the change in intensity due to absorption and scattering by ocular media and will allow accurate measurement of directional reflectivity of retinal layers. The sensitivity roll-off of the OCT signal at different depth positions were corrected and assured to be constant during the experiment. Reflections from the inner limiting membrane (ILM)/nerve fiber layer (NFL), external limiting membrane (ELM), inner-outer segment (IS/OS) junction, and Bruch's membrane (BrM)/retinal pigment epithelium (RPE) with respect to the angle of incidence were 

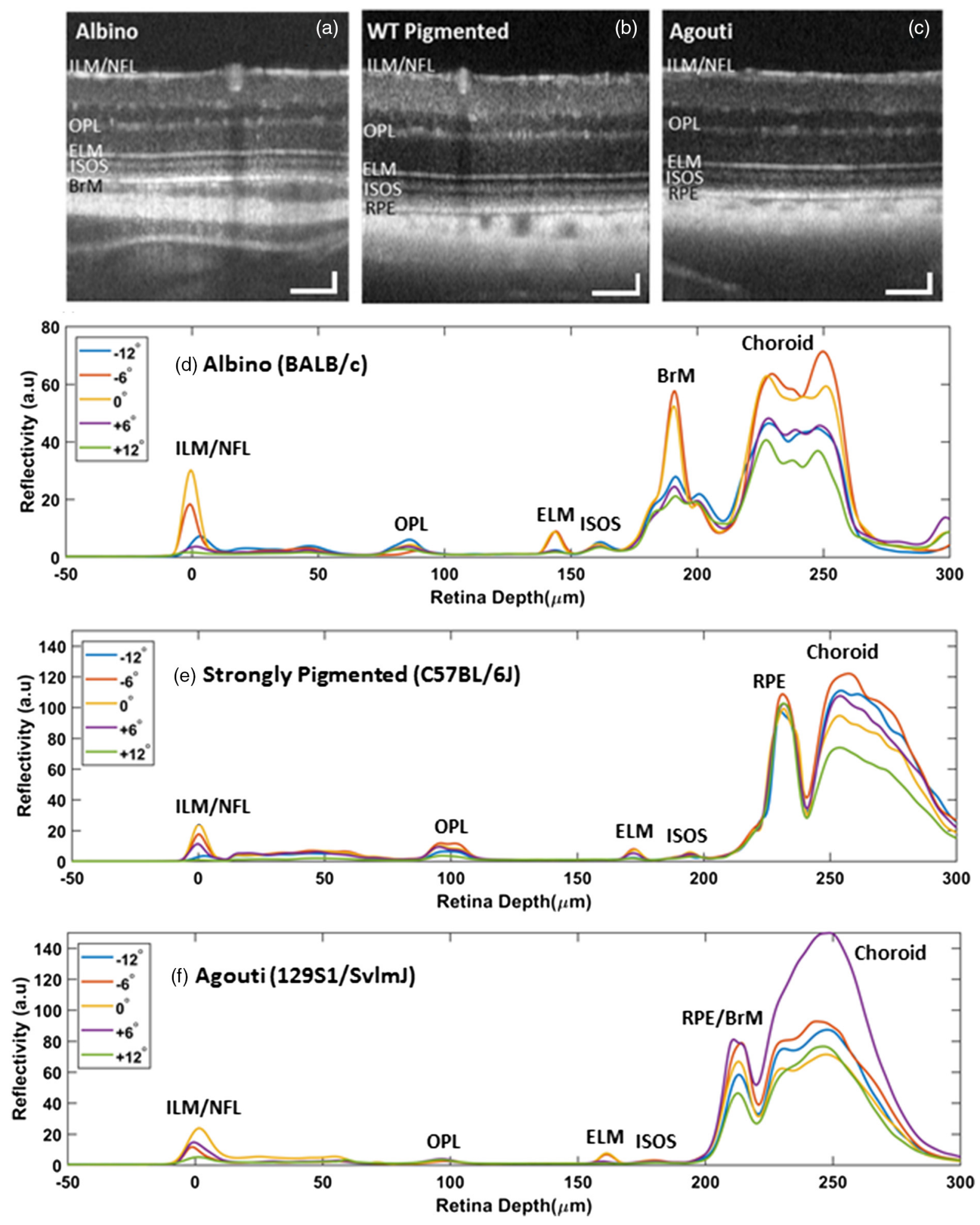

Fig. 2 (a)-(c) Representative B-scans (log scale) from different mice strains for a scan at the zero-degree incident angle. (Contrast enhancement is applied to all images for better visualization.) (d)-(f) Averaged A-scans (linear scale) generated for different angles of incidence. Horizontal scale bar: $100 \mu \mathrm{m}$. Vertical scale bar: $50 \mu \mathrm{m}$.

measured from their respective averaged A-scan profiles. The incident angle corresponding to each pupil position was calculated from the respective B-scan after converting to an aspect ratio of $1: 1$ in axial and transversal directions. The angle was measured relative to the retinal surface. Normalized reflectance as a function of incidence angle for all the major retinal layers was measured for 17 different positions and fitted with a fiveparameter Gaussian function, as given in Eq. (1). The fiveparameter model consisting of a Gaussian function and constant bias is given as 


$$
I(x)=a_{0}+a_{1} * 10^{-\rho\left[\left(x-x_{0}\right)^{2}+\left(y-y_{0}\right)^{2}\right]},
$$

where $a_{0}$ represents the intensity of the diffuse component (nondirectional scatter) in the retinal reflection. Here, $a_{1}$ and $\rho$ represent the peak intensity and directionality, respectively. Here, $x_{0}$ and $y_{0}$ represent the shift in the position from the geometric pupil center of the dilated eye.

PRISM (Version 7.02; GraphPad Software, La Jolla, California) was used for statistical analysis. An analysis of variance (ANOVA) was performed to evaluate whether there were significant differences between the reflectivity parameters across different mice strains. Differences with a $p$-value of 0.05 or less were considered statistically significant.

\section{Results and Discussion}

Representative OCT B-scans of albino, strongly pigmented, and agouti mice are shown in Figs. 2(a)-2(c), respectively. Averaged A-scans (linear scale) corresponding to different angles of incidence for albino, strongly pigmented, and agouti mice are shown in Figs. 2(d)-2(f). The ILM/NFL is unambiguously present and high scattering in the retinas of all three mouse strains, and is used for cross-strain alignment of the A-scans. The inner plexiform layer (IPL) and outer plexiform layer (OPL) appear as bright bands due to increased scattering by neuronal synapses, whereas the inner nuclear layer (INL) and ONL are relatively low-scattering layers due to watery nature of the cell bodies. A thin reflective band distal to the ONL corresponds to
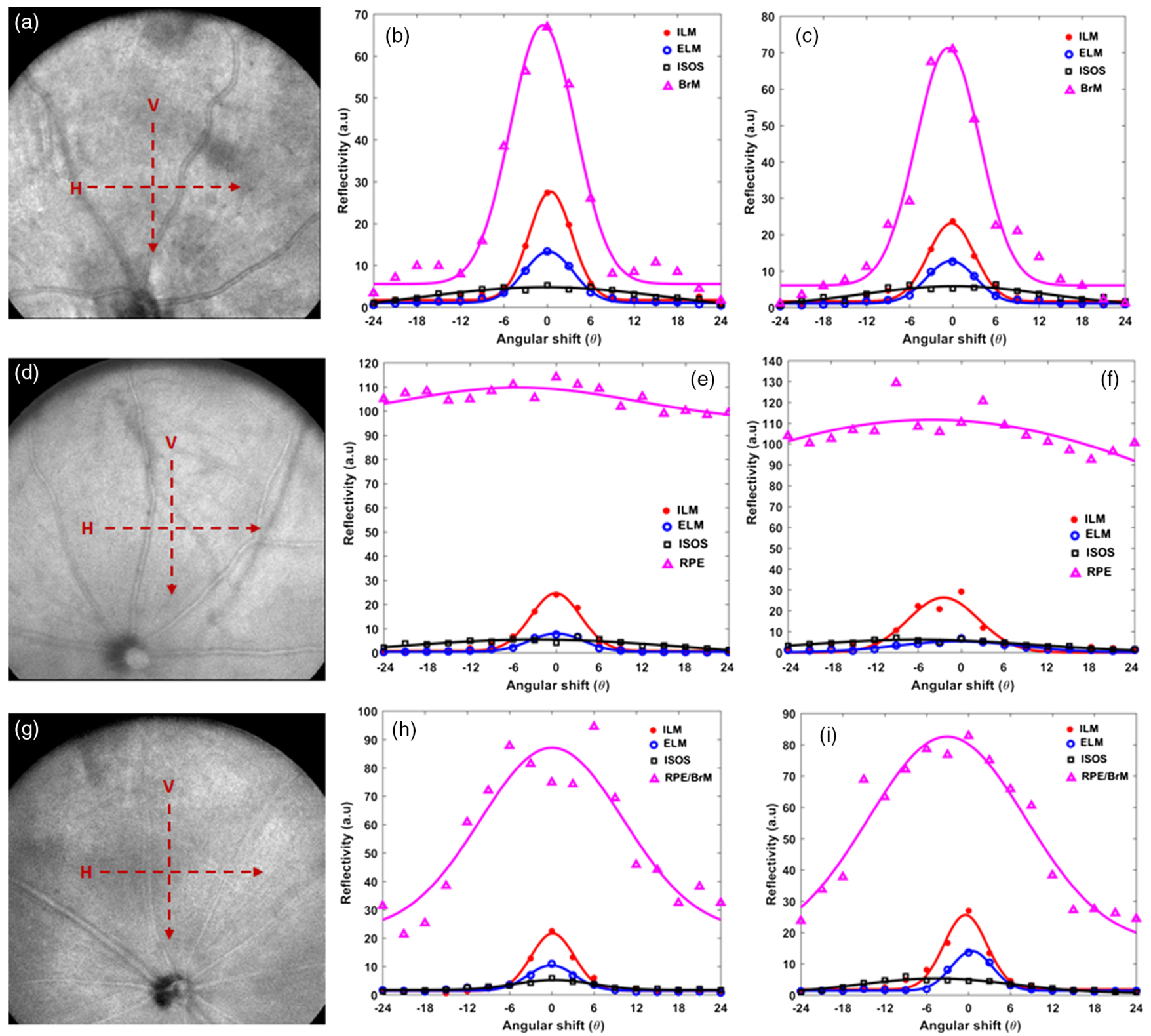

Fig. 3 OCT fundus images and directional reflectivity profiles of an albino (Balb/c) mouse, a strongly pigmented (C57BL/6J) mouse, and an agouti (129S1/SvImJ) mouse. (a), (d), and (g) "En face" OCT fundus images of albino, strongly pigmented and agouti mice, respectively. Red arrows show the location and direction of OCT scanning in $H$ and $V$ direction. (b), (e), and (h) Angular-dependent average reflectivity profile for four brightest retina layers measured along $H$. (c), (f), and (i) Angular-dependent average reflectivity profile for four brightest retina layers measured along $V$ direction. 
the external limiting membrane (ELM). A somewhat diffuse reflective band, posterior to the ELM, represents the junction between the photoreceptor IS and OS, mostly rods in the mouse retina.

The high reflectance of this layer is caused by the abrupt transition in refractive index from the IS to the OS. ${ }^{37}$ In the case of pigmented and agouti mice, there is a thick bright band posterior to the photoreceptor IS/OS junction, representing scattering from the melanin-containing RPE cells. In albino mice, which lack melanin in RPE cells, a thin highly reflective band corresponding to Bruch's membrane (BrM) appears posterior to IS/OS. The mouse retina layer assignments presented here are based on our understanding of the cellular origins of light scattering in the human retina presented by Jonnal et al. ${ }^{38,39}$ and correlations with mouse retinal histology.

Figure 3 shows directional reflectivity profiles measured for four major retinal layers of three mice strains. Figures 3(a), $3(\mathrm{~d})$, and 3(g) show the en face projections of OCT fundus volume of an albino mouse, agouti, and strongly pigmented mice, respectively. The dashed red lines on the OCT en face image fundus show the location and direction of the beam scanning. The complete dOCT images acquired from 17 entry positions in $H$ and $V$ directions for all mice strains are shown in the appendices. The acquired dOCT images show that the reflectivities of many retinal layers depend on illumination angle and therefore the visibility of these structures varies as a function of entry pupil positions (or angle of incidence). Figures 2(d)-2(f) show the representative averaged A-scan profiles indicating the variation of the reflectivity of retinal layers with the angle of incidence for albino, strongly pigmented and agouti mice, respectively. The reflectivity of major retinal layers measured for different angles of incidence and their corresponding Gaussian fitting functions are shown in Fig. 3. For all three mice strains, the reflection from the inner retinal layers ILM/NFL and ELM are highly directional, as their strength declines steeply with the angle of incidence of the OCTscanning beam on the retina. In contrast, the reflection from the IS/OS junction for all mice strains has a wider reflectivity profile as compared to ILM/NFL and ELM, possibly representing waveguiding properties of the inner segments of rod photoreceptors. ${ }^{40,41}$ Notably, in albino mice, which lack melanin pigmentation, the reflection from $\mathrm{BrM}$ is highly directional. In contrast, in a strongly pigmented mouse, reflections from the RPE are insensitive to the angle of incidence and have a flat profile similar to that reported for human RPE. ${ }^{9}$ The directional sensitivity of the reflections from the RPE/BrM complex for agouti mice is intermediate between those of strongly pigmented and albino mice.

The directional reflectivity of retinal layers in three mice strains is quantified using the directionality parameter $(\rho)$ of Eq. (1). For all mice strains, the mean and standard deviation of directionality is calculated from four mice samples (see Table 1). From the table, it is evident that the ILM/NFL and ELM exhibit relatively higher directional scattering compared to IS/OS and RPE. Furthermore, the directionality measured for ILM/NFL, ELM, and IS/OS is similar and does not statistically differ among the three mice strains $(p=0.45)$. The directionality of RPE shows marked changes among the three mice strains $(p=0.01)$. The directionality is found to be higher for albino mice and gradually reduced in agouti and highly pigmented mice. The reflections from the RPE are largely insensitive in highly pigmented mice and the directional scattering between strains shows that directionality increases with decreases in melanin concentrations in RPE.

A scatterplot of peak reflectivity versus directionality of the retinal layers of the three mice strains is given in Fig. 4. The RPE in highly pigmented mice shows increased reflectivity and reduced directionality compared to albino and agouti mice. The gradual increase in reflectivity of RPE in agouti and strongly pigmented mice relative to albino is due to the presence of melanosomes in the RPE. Here, we did not independently validate the relative amounts of melanin in the three mice strains used in this study. However, the lack of melanin pigmentation in albino (BALB/c) mice is well known and widely reported. Issa et al. ${ }^{16,42}$ have reported the difference in the melanin pigmentation levels in agouti (129S1/SvlmJ) and strongly pigmented (C57BL/6J) mice using 790-nm near-infrared fluorescence (NIRF) imaging. They found the melanosome concentration to be nearly half in agouti as compared to strongly pigmented (C57BL/6J) mice. The dominant scatterers in the pigmented RPE have been identified as the melanin granules

Table 1 Average directionality $(\rho)$ and standard deviations for three mice strains.

\begin{tabular}{|c|c|c|c|c|c|c|}
\hline \multirow[b]{2}{*}{ Layers } & \multicolumn{2}{|c|}{ Albino } & \multicolumn{2}{|c|}{ Agouti } & \multicolumn{2}{|c|}{ Strongly Pigmented } \\
\hline & $H$ & v & $H$ & V & $H$ & v \\
\hline \multirow[t]{2}{*}{ ILM/NFL } & 0.251 & 0.210 & 0.290 & 0.358 & 0.214 & 0.200 \\
\hline & $( \pm 0.035)$ & $( \pm 0.022)$ & $( \pm 0.015)$ & $( \pm 0.011)$ & $( \pm 0.021)$ & $( \pm 0.025)$ \\
\hline \multirow[t]{2}{*}{ ELM } & 0.192 & 0.189 & 0.258 & 0.293 & 0.187 & 0.193 \\
\hline & $( \pm 0.015)$ & $( \pm 0.022)$ & $( \pm 0.035)$ & $( \pm 0.021)$ & $( \pm 0.047)$ & $( \pm 0.035)$ \\
\hline \multirow[t]{2}{*}{ IS/OS } & 0.011 & 0.012 & 0.021 & 0.014 & 0.031 & 0.020 \\
\hline & $( \pm 0.008)$ & $( \pm 0.005)$ & $( \pm 0.004)$ & $( \pm 0.002)$ & $( \pm 0.007)$ & $( \pm 0.005)$ \\
\hline \multirow[t]{2}{*}{ RPE/BrM } & 0.151 & 0.121 & 0.043 & 0.054 & 0.001 & 0.002 \\
\hline & $( \pm 0.003)$ & $( \pm 0.005)$ & $( \pm 0.008)$ & $( \pm 0.004)$ & $( \pm 0.001)$ & $( \pm 0.001)$ \\
\hline
\end{tabular}




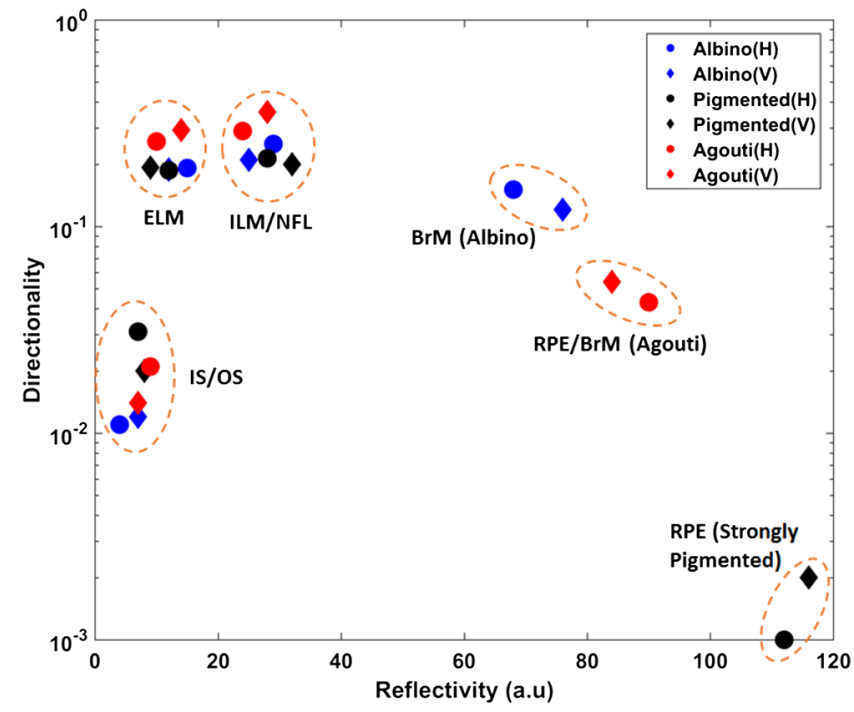

Fig. 4 Scatterplot showing both directionality and reflectivity of retinal layers.

(melanosomes). ${ }^{18,43}$ The scattering nature of a melanin granule depends on its shape and effective size relative to the wavelength of the light used for imaging. The average size of the melanin granules varies from $600 \mathrm{~nm}$ to $1 \mu \mathrm{m}$ in diameter, resulting in a size (diffraction) parameter $x=2 \pi r / \lambda$ in the four to six range (for $\lambda=1.05 \mu \mathrm{m}$ ), clearly placing melanosome scattering in the Mie scattering regime range. ${ }^{44}$ In general, we expect that change in size and shape, not just the number of scatterers, could also be studied using our dOCT method. Based on this average size of melanosomes known from other studies, we conclude that they are responsible for strong scattering from the RPE layer. The directionality and relative reflectivity of the other layers were found to be similar for all mice strains under the same experimental conditions, confirming that indeed the melanin concentration in RPE is responsible for the observed variation in scattering from this layer.

\section{Conclusion}

The directional reflectivities of the major scattering layers in retinas of mice with different melanin concentration levels were investigated with a dOCT system. The magnitude and directional scattering from the RPE depend on the melanin concentration in RPE cells. RPE with higher melanin concentration showed increased scattering but reduced directionality (Fig. 4). In other words, the directionality in the RPE/BrM complex increased with decreases in RPE melanin concentration. Highly reflective and melanin-free layers such as ILM/NFL, ELM, and BrM (in albino mice) were found to be highly sensitive to the angle of illumination, but as expected, they did not vary between mice strains of different melanin concentrations. We anticipate that the capability of directional reflectivity of RPE to detect the relative changes in melanin pigmentation in RPE could be a powerful tool/biomarker for the investigation of RPE in vivo. The proposed methodology could be extended for the investigation of pathological eyes with arbitrary RPE shape, albeit, a local change in RPE layer orientation would require the local calculation of directionality rather than a global one. Further studies are needed to establish age and diseaserelated changes in melanin concentration in vivo. OCT systems with the higher axial resolution and the different spectral band should be explored to enhance the sensitivity of this technique. OCT system with higher axial resolution would allow axial localization of the melanin granules within the RPE cells to allow probing of the subcellular morphology of these highly specialized cells. ${ }^{44}$

\section{Appendices}

The directional OCT images have been acquired by varying the position of the entrance pupil (imaging beam) with respect to the dilated mouse pupil using motorized linear stages in horizontal $(\mathrm{H})$ and Vertical $(\mathrm{V})$ directions. The representative directional OCT images along $\mathrm{H}$ and $\mathrm{V}$ directions for albino (Figs. 5 and 6), highly pigmented (Figs. 7 and 8), and mildly pigmented agouti mice (Figs. 9 and 10) are presented below. The conceptual schematic of the dilated pupil of mouse eye (blue circle) with different positions of the imaging beam (red dots) during single directional imaging session are presented for each scanning series.
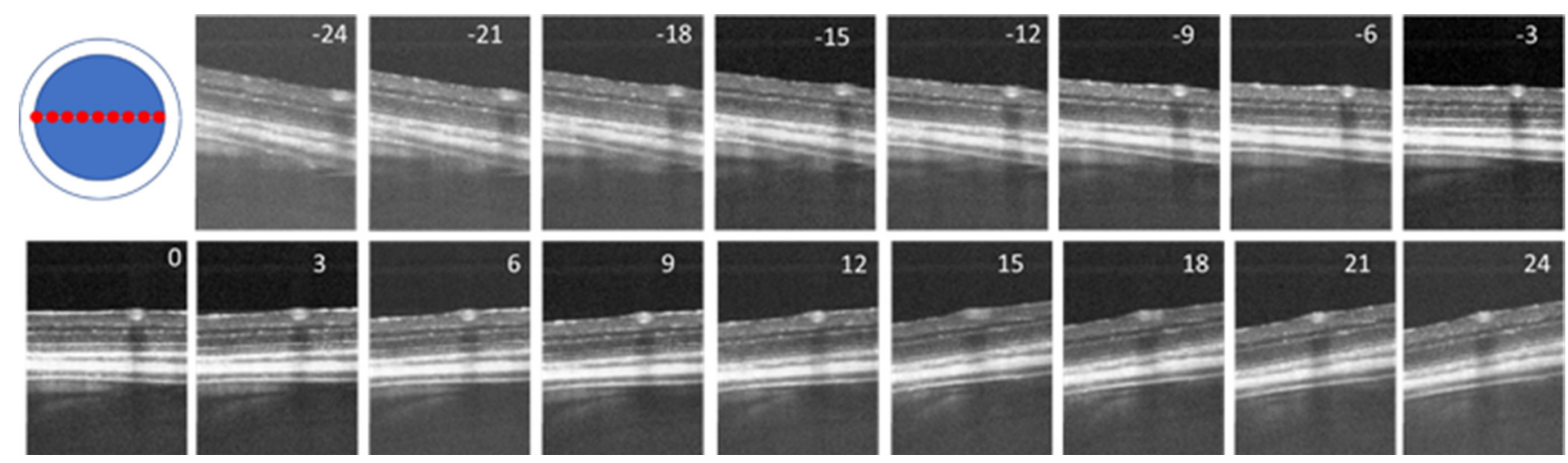

Fig. 5 Averaged $B$-scans (log scale) acquired from 17 beam entry positions in $H$ direction for Albino (Balb/c) mice. The numbers shown in the images represent the angle of tilt of the images. 
Albino (Balb/c): $V$ direction

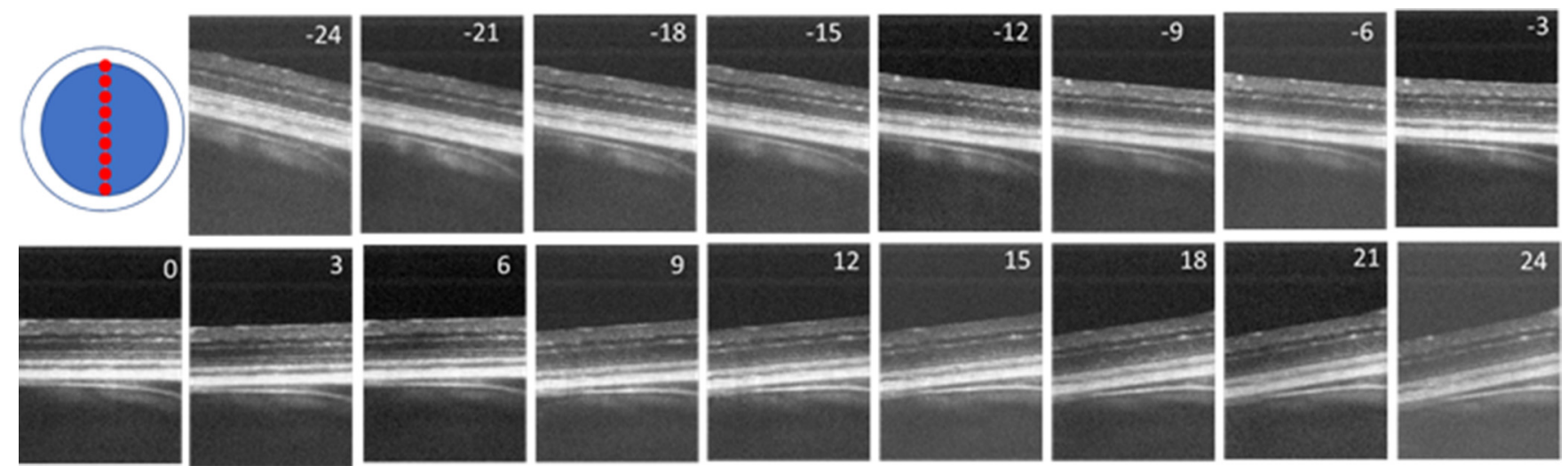

Fig. 6 Averaged $B$-scans (log scale) acquired from 17 beam entry positions in $V$ direction for Albino (Balb/c) mice. The numbers shown in the images represent the angle of tilt of the images.

Strongly Pigmented (C57BL/6J): $H$ direction

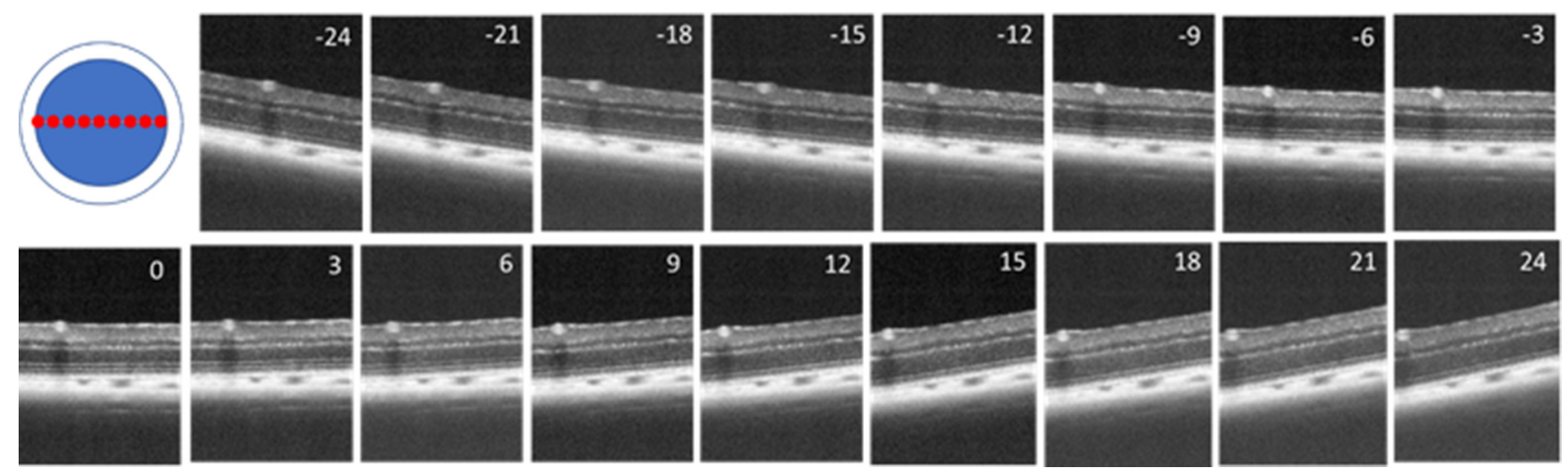

Fig. 7 Averaged $B$-scans (log-scale) acquired from 17 beam entry positions in $H$ direction for strongly pigmented (C57BL/6J) mice. The numbers shown in the images represent the angle of tilt of the images.

Strongly Pigmented (C57BL/6J): $V$ direction
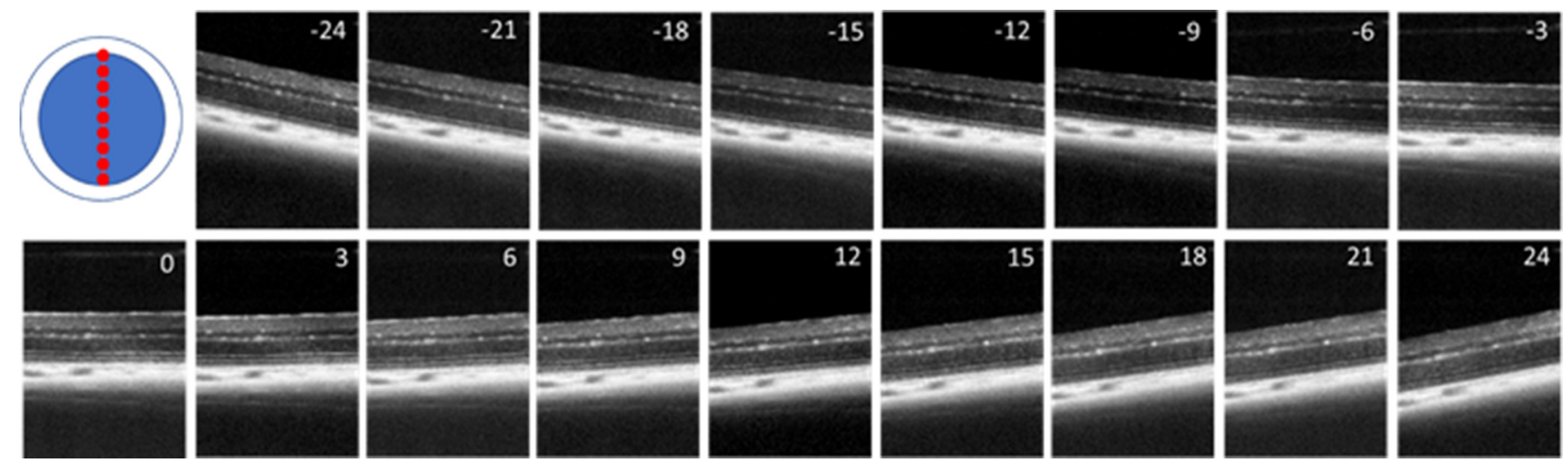

Fig. 8 Averaged $B$-scans (log scale) acquired from 17 beam entry positions in $V$ direction for strongly pigmented (C57BL/6J) mice. The numbers shown in the images represent the angle of tilt of the images. 
Agouti (129S1/SvlmJ): $H$ direction
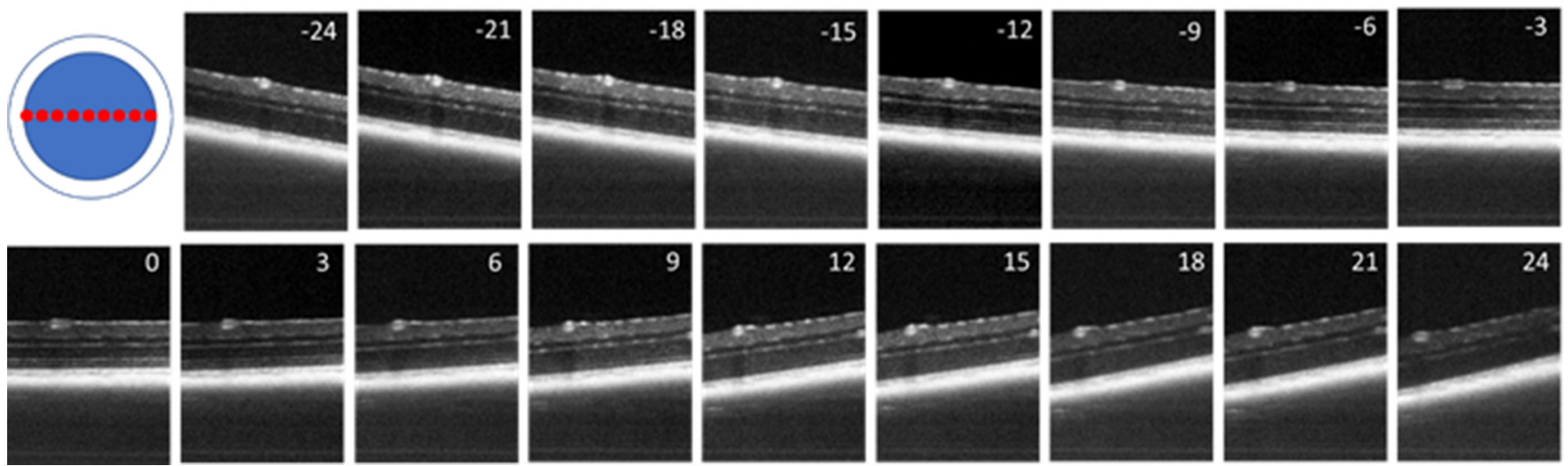

Fig. 9 Averaged $B$-scans (log scale) acquired from 17 beam entry positions in $H$ direction for agouti (129S1/SvImJ) mice. The numbers shown in the images represent the angle of tilt of the images.

\section{Agouti (129S1/SvlmJ): $V$ direction}
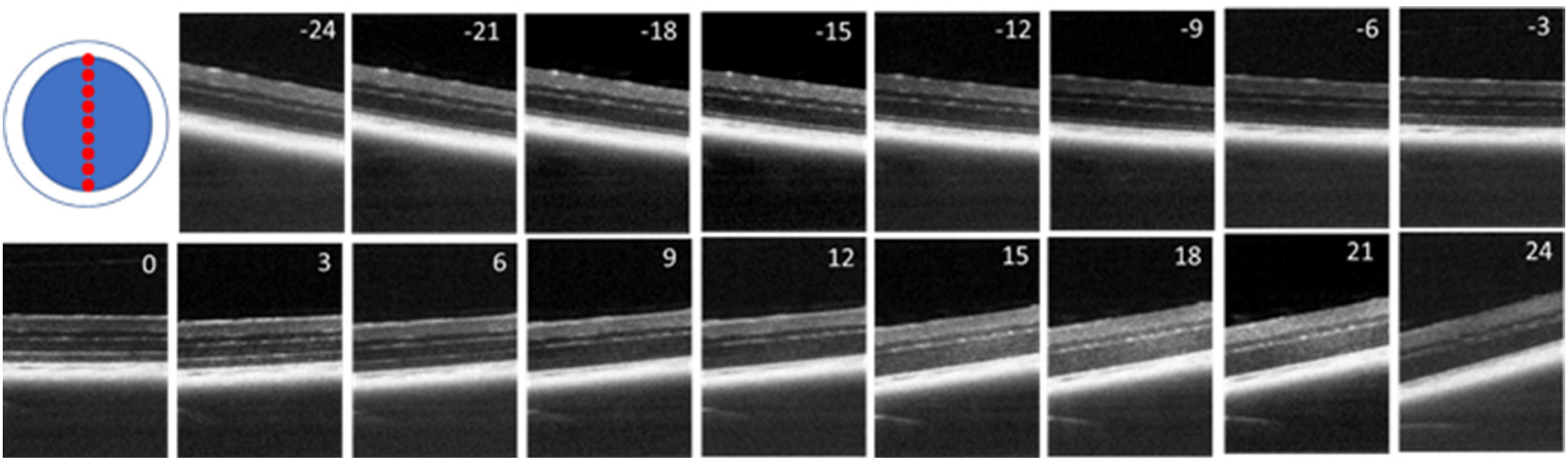

Fig. 10 Averaged $B$-scans (log scale) acquired from 17 beam entry positions in $V$ direction for agouti $(129 \mathrm{~S} 1 / \mathrm{SvlmJ})$ mice. The numbers shown in the images represent the angle of tilt of the images.

\section{Disclosures}

The authors declare no conflict of interest.

\section{Acknowledgments}

Authors would like to acknowledge their funding sources: NSF I/UCRC CBSS Grant, NIH Grant Nos. EY026556 and EY012576 (NEI Core Grant), and Barr Retina Research Foundation gift to UC Davis Department of Ophthalmology. They thank Professor Ravi S. Jonnal (UC Davis Vision Science and Advanced Retinal Imaging Laboratory) and Marinko V. Sarunic (Simon Fraser University, Canada) for their fruitful discussions and support.

\section{References}

1. B. Cense et al., "Ultrahigh-resolution high-speed retinal imaging using spectral-domain optical coherence tomography," Opt. Express 12(11), 2435-2447 (2004).

2. M. Wojtkowski et al., "Three-dimensional retinal imaging with high-speed ultrahigh-resolution optical coherence tomography," Ophthalmology 112(10), 1734-1746 (2005).
3. S. K. Gardiner et al., "Changes in retinal nerve fiber layer reflectance intensity as a predictor of functional progression in glaucoma," Invest. Ophthalmol. Visual Sci. 57(3), 1221-1227 (2016).

4. M. E. Pons et al., "Assessment of retinal nerve fiber layer internal reflectivity in eyes with and without glaucoma using optical coherence tomography," Arch. Ophthalmol. 118(8), 1044-1047 (2000).

5. X.-R. Huang et al., "Reflectance decreases before thickness changes in the retinal nerve fiber layer in glaucomatous retinas," Invest. Ophthalmol. Visual Sci. 52(9), 6737-6742 (2011).

6. R. W. Knighton and C. Qian, "An optical model of the human retinal nerve fiber layer: implications of directional reflectance for variability of clinical measurements," J. Glaucoma 9(1), 56-62 (2000).

7. B. J. Lujan et al., "Revealing Henle's fiber layer using spectral domain optical coherence tomography," Invest. Ophthalmol. Visual Sci. 52(3), 1486-1492 (2011).

8. K. K. Tong et al., "Directional optical coherence tomography reveals reliable outer nuclear layer measurements," Optom. Vision Sci. 93(7), 714-719 (2016).

9. W. Gao et al., "Measuring retinal contributions to the optical StilesCrawford effect with optical coherence tomography," Opt. Express 16(9), 6486-6501 (2008).

10. T. Sarna, "New trends in photobiology: properties and function of the ocular melanin - a photobiophysical view," J. Photochem. Photobiol. B 12(3), 215-258 (1992). 
11. Z. Wang, J. Dillon, and E. R. Gaillard, "Antioxidant properties of melanin in retinal pigment epithelial cells," Photochem. Photobiol. 82(2), 474-479 (2006).

12. S. Peters et al., "Melanin protects choroidal blood vessels against light toxicity," Z. Naturforsch. C 61(5-6), 427-433 (2006).

13. L. Feeney, "Lipofuscin and melanin of human retinal pigment epithelium. Fluorescence, enzyme cytochemical, and ultrastructural studies," Invest. Ophthalmol. Visual Sci. 17(7), 583-600 (1978).

14. J. J. Weiter et al., "Retinal pigment epithelial lipofuscin and melanin and choroidal melanin in human eyes," Invest. Ophthalmol. Visual Sci. 27(2), 145-52 (1986).

15. S. Y. Schmidt and R. D. Peisch, "Melanin concentration in normal human retinal pigment epithelium. Regional variation and age-related reduction," Invest. Ophthalmol. Visual Sci. 27(7), 1063-1067 (1986).

16. C. N. Keilhauer and F. O. C. Delori, "Near-infrared autofluorescence imaging of the fundus: visualization of ocular melanin," Invest. Ophthalmol. Visual Sci. 47(8), 3556-3564 (2006).

17. U. Kellner, S. Kellner, and S. Weinitz, "Fundus autofluorescence $(488 \mathrm{~nm})$ and near-infrared autofluorescence $(787 \mathrm{~nm})$ visualize different retinal pigment epithelium alterations in patients with age-related macular degeneration," Retina 30(1), 6-15 (2010).

18. M. A. Wilk et al., "The effect of retinal melanin on optical coherence tomography images," Transl. Vision Sci. Technol. 6(2), 8 (2017).

19. B. Baumann et al., "Polarization sensitive optical coherence tomography of melanin provides intrinsic contrast based on depolarization," Biomed. Opt. Express 3(7), 1670-1683 (2012).

20. M. Pircher, C. K. Hitzenberger, and U. Schmidt-Erfurth, "Polarization sensitive optical coherence tomography in the human eye," Prog. Retinal Eye Res. 30(6), 431-451 (2011).

21. C. Ahlers et al., "Imaging of the retinal pigment epithelium in agerelated macular degeneration using polarization-sensitive optical coherence tomography," Invest. Ophthalmol. Visual Sci. 51(4), 2149-2157 (2010).

22. S. Azuma et al., "Pixel-wise segmentation of severely pathologic retinal pigment epithelium and choroidal stroma using multi-contrast Jones matrix optical coherence tomography," Biomed. Opt. Express 9(7), 2955-2973 (2018).

23. M. Lapierre-Landry et al., "In vivo photothermal optical coherence tomography of endogenous and exogenous contrast agents in the eye," Sci. Rep. 7(1), 9228 (2017).

24. M. Lapierre-Landry et al., "Imaging melanin distribution in the zebrafish retina using photothermal optical coherence tomography," Transl. Vision Sci. Technol. 7(5), 4-4 (2018).

25. L. T. Perelman et al., "Observation of periodic fine structure in reflectance from biological tissue: a new technique for measuring nuclear size distribution," Phys. Rev. Lett. 80(3), 627-630 (1998).

26. J. W. Pyhtila, R. N. Graf, and A. Wax, "Determining nuclear morphology using an improved angle-resolved low coherence interferometry system," Opt. Express 11(25), 3473-3484 (2003).

27. J. R. Mourant et al., "Mechanisms of light scattering from biological cells relevant to noninvasive optical-tissue diagnostics," Appl. Opt. 37(16), 3586-3593 (1998).

28. M. A. Choma, C. Yang, and J. A. Izatt, "Instantaneous quadrature lowcoherence interferometry with $3 \times 3$ fiber-optic couplers," Opt. Lett. 28(22), 2162-2164 (2003).

29. R. K. Meleppat et al., "Quantitative optical coherence microscopy for the in-situ investigation of the biofilm," J. Biomed. Opt. 21(12), 127002 (2016).

30. K. M. Ratheesh, L. K. Seah, and V. M. Murukeshan, "Spectral phasebased automatic calibration scheme for swept source-based optical coherence tomography systems," Phys. Med. Biol. 61(21), 7652-7663 (2016).

31. R. K. Meleppat, M. V. Matham, and L. K. Seah, "An efficient phase analysis-based wavenumber linearization scheme for swept source optical coherence tomography systems," Laser Phys. Lett. 12(5), 055601 (2015).
32. P. Thevenaz, U. E. Ruttimann, and M. Unser, "A pyramid approach to subpixel registration based on intensity," IEEE Trans. Image Process. 7(1), 27-41 (1998).

33. R. K. Meleppat et al., "Investigation of the effect of directional (off-axis) illumination on the reflectivity of retina layers in mice using sweptsource optical coherence tomography," Proc. SPIE 10474, 104742B (2018).

34. D. V. Norren and J. J. Vos, "Spectral transmission of the human ocular media," Vision Res. 14(11), 1237-1244 (1974).

35. G. J. Van Blokland and S. C. Verhelst, "Corneal polarization in the living human eye explained with a biaxial model," J. Opt. Soc. Am. A 4(1), 82-90 (1987).

36. X. Zhang, A. Bradley, and L. N. Thibos, "Achromatizing the human eye: the problem of chromatic parallax," J. Opt. Soc. Am. A 8(4), 686-691 (1991).

37. P. Zhang et al., "In vivo optophysiology reveals that G-protein activation triggers osmotic swelling and increased light scattering of rod photoreceptors," Proc. Natl. Acad. Sci. U.S.A. 114(14), E2937-E2946 (2017).

38. R. S. Jonnal et al., "The properties of outer retinal band three investigated with adaptive-optics optical coherence tomography," Invest. Ophthalmol. Visual Sci. 58(11), 4559-4568 (2017).

39. R. S. Jonnal et al., "The cellular origins of the outer retinal bands in optical coherence tomography images," Invest. Ophthalmol. Visual Sci. 55(12), 7904-7918 (2014).

40. J. F. L. Tobey and J. M. Enoch, "Directionality and waveguide properties of optically isolated rat rods," Invest. Ophthalmol. Visual Sci. 12(12), 873-880 (1973).

41. M. Alpern, C. C. Ching, and K. Kitahara, "The directional sensitivity of retinal rods," J. Physiol. 343, 577-592 (1983).

42. P. C. Issa et al., "Fundus autofluorescence in the Abca4-/- mouse model of Stargardt disease-correlation with accumulation of A2E, retinal function, and histology," Invest. Ophthalmol. Visual Sci. 54(8), 5602-5612 (2013).

43. A. Berger et al., "Spectral-domain optical coherence tomography of the rodent eye: highlighting layers of the outer retina using signal averaging and comparison with histology," PLoS One 9(5), e96494 (2014).

44. A. Pollreisz et al., "Visualizing melanosomes, lipofuscin, and melanolipofuscin in human retinal pigment epithelium using serial block face scanning electron microscopy," Exp. Eye Res. 166, 131-139 (2018).

Ratheesh K. Meleppat received his Bachelor of Technology degree in electronics and communication engineering from the University of Calicut, India, in 2006 and his Master of Technology in optoelectronics and optical communications from the University of Kerala, India, in 2011. He obtained his $\mathrm{PhD}$ in biomedical optics and imaging from Nanyang Technological University, Singapore, in 2017. Currently, he is a postdoctoral research scholar at the University of California Davis, California. He also serves as an active member of SPIE, ARVO, and IEEE Photonics Society.

Pengfei Zhang is a project scientist at the University of California Davis. He received his $\mathrm{PhD}$ in optical engineering and instrumentation from the Xi'an Jiao Tong University in 2011. His current research interests include development and application of in vivo retinal imaging systems (scanning laser ophthalmoscopy and optical coherence tomography, and their combinations with adaptive optics) for studying structural and functional changes at cellular resolution.

Robert J. Zawadzki is an associate professor of ophthalmology at UC Davis. He received BS and MS degrees in experimental physics and medical physics from the Nicolaus Copernicus University Torun, Poland, and his PhD in natural sciences from Technical University of Vienna, Austria. In 2004, he joined the UC Davis Vision Science and Advanced Retinal Imaging (VSRI) Laboratory. Since 2012, he has also been acting as managing director of UC Davis EyePod.

Biographies of the other authors are not available. 